Thomas Ihn

\title{
Electronic Quantum Transport in Mesoscopic Semiconductor Structures
}

With 90 Illustrations, 5 in Full Color 


\section{Contents}

\section{Part I Introduction to Electron Transport}

1 Electrical conductance: Historical account from Ohm to the semiclassical Drude-Boltzmann theory $\ldots \ldots \ldots \ldots \ldots \ldots \ldots \ldots, \ldots$

2 Toward the microscopic understanding of conductance on a quantum mechanical basis $\ldots \ldots \ldots \ldots \ldots \ldots \ldots \ldots \ldots \ldots \ldots$

2.1 Quantum transport in metals $\ldots \ldots \ldots \ldots \ldots \ldots \ldots \ldots \ldots \ldots \ldots \ldots$

2.2 Transistors and two-dimensional electron gases in semiconductors , 10

2.2.1 Two-dimensional electron gases in field-effect transistors. . . 10

2.2.2 Resonant tunneling in semiconductors ............. 13

2.2.3 Integer and fractional quantum Hall effect $\ldots \ldots \ldots \ldots \ldots, 14$

2.2 .4 Weak localization .......................... 15

2.3 Basic phenomena in semiconductor structures of reduced size and

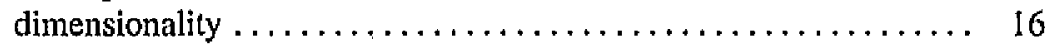

2.3.1 The Aharonov-Bohm effect and conductance fluctuations .. 16

2.3.2 Conductance quantization in semiconductor quantum point contacts $\ldots \ldots \ldots \ldots \ldots \ldots \ldots \ldots \ldots \ldots \ldots \ldots, 18$

2.3.3 Semiconductor quantum dots and artificial atoms $\ldots \ldots \ldots, 19$

Part II Conductance in Strongly Interacting and Disordered Two-Dimensional Systems

3 The concept of metals and insulators

4 Scaling theory of localization $\ldots \ldots \ldots \ldots \ldots \ldots \ldots \ldots \ldots \ldots$

5 Electron-electron interactions within the Fermi-liquid concept . . . . 29

5.1 Dephasing in diffusive two-dimensional systems $\ldots \ldots \ldots \ldots \ldots .30$

5.2 Interaction corrections to the conductivity $\ldots \ldots \ldots \ldots \ldots \ldots, 30$ 
5.2.1 Temperature-dependent screening

5.2.2 Interaction corrections due to interference of multiply scattered paths $\ldots \ldots \ldots \ldots \ldots \ldots \ldots \ldots \ldots \ldots \ldots \ldots$

5.2.3 A comprehensive theory of interaction corrections based on the Fermi liquid concept $\ldots \ldots \ldots \ldots \ldots \ldots \ldots \ldots, 34$

6 Beyond Fermi-liquid theory $\ldots \ldots \ldots \ldots \ldots \ldots \ldots \ldots \ldots \ldots$

7 Summary of disorder and interaction effects $\ldots \ldots \ldots \ldots \ldots \ldots$

8 Experiments on strongly interacting two-dimensional systems and the metal-insulator transition $\ldots \ldots \ldots \ldots \ldots \ldots \ldots \ldots \ldots \ldots \ldots$

9 Theoretical work related to the metal-insulator transition $\ldots \ldots \ldots 43$

10 Metallic behavior in p-SiGe quantum wells $\ldots \ldots \ldots \ldots \ldots \ldots \ldots \ldots$

10.1 Samples and structures $\ldots \ldots \ldots \ldots \ldots \ldots \ldots \ldots \ldots \ldots \ldots 45$

10.2 Scaling analysis, quantum phase transition, and heating effects ... 47

10.3 Magnetoresistance measurements . . . . . . . . . . . . . . . 49

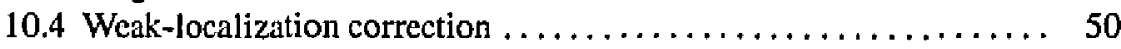

10.5 Interaction corrections to the conductivity: multiple impurity

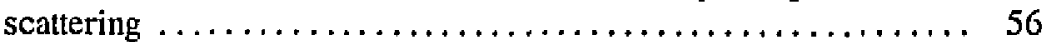

10.6 Interaction corrections of the Drude conductivity due to $T$-dependent screening $\ldots \ldots \ldots \ldots \ldots \ldots \ldots \ldots \ldots \ldots \ldots, 58$

10.7 Reentrant insulating behavior $\ldots \ldots \ldots \ldots \ldots \ldots \ldots \ldots \ldots \ldots \ldots 61$

10.8 Parallel magnetic field $\ldots \ldots \ldots \ldots \ldots \ldots \ldots \ldots \ldots \ldots \ldots \ldots, 61$

10.9 Discussion of the results and conclusions $\ldots \ldots \ldots \ldots \ldots \ldots \ldots, 62$

Part III Electron Transport through Quantum Dots and Quantum Rings

11 Introduretion to electron transport through quantum dots . . . . . . 67

11.1 Resonant tunneling and the quantization of the particle number on weakly coupled islands........................ 67

11.2 Quantum dot states: from a general hamiltonian to the constant-interaction model $\ldots \ldots \ldots \ldots \ldots \ldots \ldots \ldots \ldots \ldots, 70$

11.3 Transport through quantum dots $\ldots \ldots \ldots \ldots \ldots \ldots \ldots \ldots \ldots \ldots, 76$

11.3.1 Coulomb-blockade oscillations ................ 76

11.3.2 Coulomb-blockade diamonds $\ldots \ldots \ldots \ldots \ldots \ldots \ldots \ldots, 78$

11.3.3 Conductance peak line shape at finite temperatures ...... 80

11.4 Beyond the constant-interaction model................. 84

12 Energy spectra of quantum rings $\ldots \ldots \ldots \ldots \ldots \ldots \ldots \ldots \ldots$

12.1 Introduction to quantum rings $\ldots \ldots \ldots \ldots \ldots \ldots \ldots \ldots \ldots \ldots, 87$

12.2 Samples and structures $\ldots \ldots \ldots \ldots \ldots \ldots \ldots \ldots \ldots \ldots \ldots \ldots \ldots, 8 \ldots \ldots$

12.3 Magnetolransport measurements on a quantum ring .......... 89 
12.4 Interpretation within the constant-interaction model .......... 91

12.5 One-dimensional ring model $\ldots \ldots \ldots \ldots \ldots \ldots \ldots \ldots \ldots \ldots, 92$

12.6 Ring with finite width $\ldots \ldots \ldots \ldots \ldots \ldots \ldots \ldots \ldots \ldots \ldots \ldots, \ldots 4$

12.7 Experimental single-particle level spectrum............. 96

12.8 Effects of broken symmetry $\ldots \ldots \ldots \ldots \ldots \ldots \ldots \ldots \ldots \ldots, 98$

12.9 Interaction effects and spin-pairing $\ldots \ldots \ldots \ldots \ldots \ldots \ldots \ldots, 99$

12.10Coulomb-blockade in a Sinai billiard ................ 104

12.11 Relation of the ring spectra to persistent currents $\ldots \ldots \ldots \ldots \ldots 106$

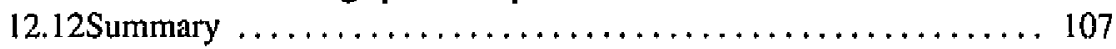

13 Spin filling in quantum dots $\ldots \ldots \ldots \ldots \ldots \ldots \ldots \ldots \ldots$

13.1 Introduction to spins in quantum dots $\ldots \ldots \ldots \ldots \ldots \ldots \ldots \ldots 109$

13.2 Samples and structures $\ldots \ldots \ldots \ldots \ldots \ldots \ldots \ldots \ldots \ldots \ldots \ldots 112$

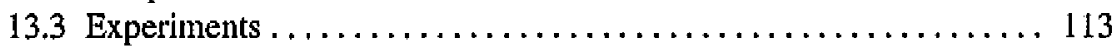

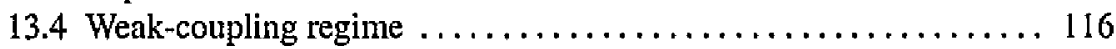

13.5 Intermediate-coupling regime $\ldots \ldots \ldots \ldots \ldots \ldots \ldots \ldots \ldots \ldots 119$

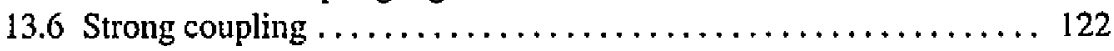

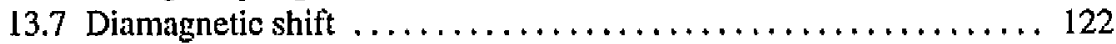

13.8 Discussion of the results $\ldots \ldots \ldots \ldots \ldots \ldots \ldots \ldots \ldots \ldots \ldots \ldots \ldots$

13.9 Conclusions $\ldots \ldots \ldots \ldots \ldots \ldots \ldots \ldots \ldots \ldots \ldots \ldots \ldots \ldots \ldots \ldots \ldots$

\section{Part IV Local Spectroscopy of Semiconductor Nanostructures}

14 Instrumentation: Scanning force microscopes for cryogenic

temperatures and magnetic fields $\ldots \ldots \ldots \ldots \ldots \ldots \ldots \ldots \ldots \ldots \ldots$

14.1 Introduction: low-temperature scanning force microscopes ...... 131

14.2 Design criteria for a low-temperature scanning force microscope for the investigation of semiconductor nanostructures $\ldots \ldots \ldots \ldots 131$

14.3 A scanning force microscope operated in a ${ }^{3} \mathrm{He}$ system . . . . . . 133

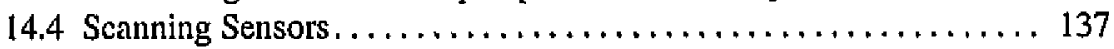

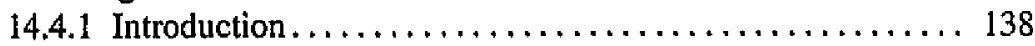

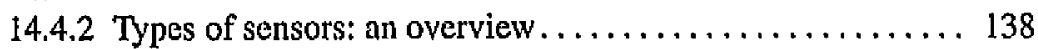

14.4.3 Piezoelectric tuning fork sensors $\ldots \ldots \ldots \ldots \ldots \ldots \ldots \ldots 141$

14.5 Electronics for a high-Q tuning fork sensor $\ldots \ldots \ldots \ldots \ldots \ldots \ldots 150$

14.5.1 Tuning fork admittance and frequency demodulation ..... 150

14.5.2 Frequency detection with a phase-locked loop . . . . . . 154

14.5.3 Frequency shift and tip-sample interaction ........... 158

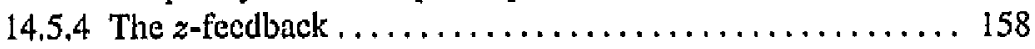

14.5.5 About feedback parameters ................... 160

14.6 Force-distance studies on HOPG with piezoelectric tuning forks at

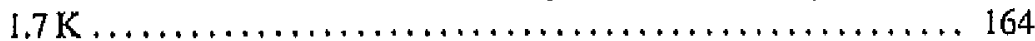


15 Local investigation of a two-dimensional electron gas with an SFM at cryogenic temperatures $\ldots \ldots \ldots \ldots \ldots \ldots \ldots \ldots \ldots \ldots \ldots \ldots, \ldots, \ldots$

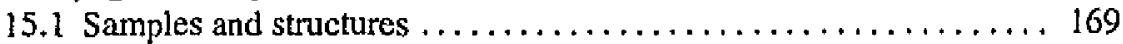

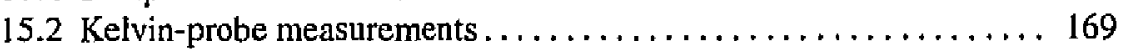

15.3 General electrostatic consideration ................. 173

15.4 Plate capacitor model $\ldots \ldots \ldots \ldots \ldots \ldots \ldots \ldots \ldots \ldots \ldots \ldots$

16 Local investigation of edge channels . . . . . . . . . . . . . . 179

16.1 Brief introduction to edge channels . . . . . . . . . . . . . 179

16.2 Scanning probe experiments $\ldots \ldots \ldots \ldots \ldots \ldots \ldots \ldots \ldots \ldots \ldots$

16.2.1 Scanning SET measurements $\ldots \ldots \ldots \ldots \ldots \ldots \ldots \ldots \ldots 181$

16.2 .2 Scanned potential microscopy $\ldots \ldots \ldots \ldots \ldots \ldots \ldots \ldots \ldots 182$

16.2.3 Subsurface charge accumulation imaging ........... 184

16.2.4 Local modification of inter-edgc-channel tunneling with a scanning force microscope $\ldots \ldots \ldots \ldots \ldots \ldots \ldots \ldots, 185$

17 Scanning gate measurements on a quantum wire . . . . . . . . . . 191

17.1 Introduction to scanning gate measurements on mesoscopic systems 191

17.2 Samples and structures $\ldots \ldots \ldots \ldots \ldots \ldots \ldots \ldots \ldots \ldots \ldots . \ldots 19$

17.3 Results of low-temperature scanning gate measurements on a quantum wire $\ldots \ldots \ldots \ldots \ldots \ldots \ldots \ldots \ldots \ldots \ldots \ldots \ldots \ldots$

17.4 Modeling scanning gate measurements: Classical and quantum

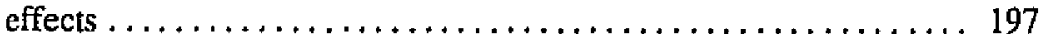

17.4.1 Classical billiard model . . . . . . . . . . . . . . . . . . . 198

17.4.2 Quantum description of scattering in wires . . . . . . . 200

A Formal solution of the electrostatic problem with Green's functions . . 207

A.1 The electrostatic problem ....................... 207

A.2 Formal solution with Green's functions $\ldots \ldots \ldots \ldots \ldots \ldots \ldots \ldots 208$

A.3 Induced charges on the electrodes ..................... 209

A.4 Total electrostatic energy of the system $\ldots \ldots \ldots \ldots \ldots \ldots \ldots \ldots 210$

A.5 Force gradient acting on an electrode $\ldots \ldots \ldots \ldots \ldots \ldots \ldots \ldots, 210$

B Screened addition energy of an electron to a quantum ring . . . . . . 211

C Scattering in quantum wires $\ldots \ldots \ldots \ldots \ldots \ldots \ldots \ldots \ldots \ldots \ldots \ldots \ldots$

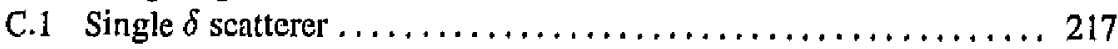

C.2 Multiple $\delta$ scatterers ........................... 219

C.3 Delta scatterer with finite extent in $y$-direction $\ldots \ldots \ldots \ldots \ldots \ldots 220$

C.4 Scatterer with finite extent in $y$-direction and rectangular shape in

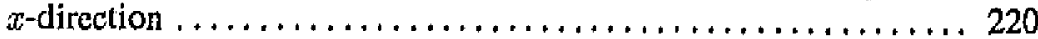

D Response of a harmonic oscillator to a resonance frequency step . . . , 223

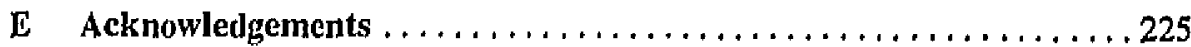




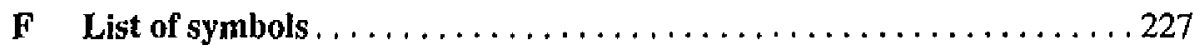

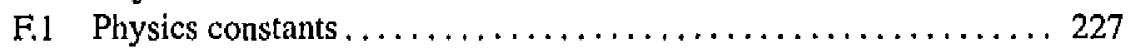

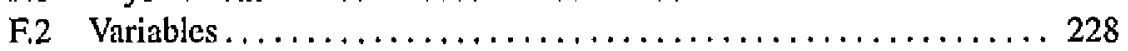

F.3 Special functions $\ldots \ldots \ldots \ldots \ldots \ldots \ldots \ldots \ldots \ldots \ldots \ldots \ldots$

References . . . . . . . . . . . . . . . . . . . . . 239

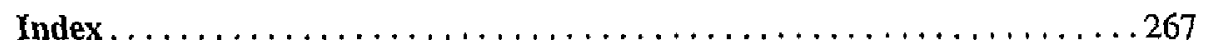

